\title{
EL RÉGIMEN DE PROTECCIÓN JURÍdICA DE LAS OBTENCIONES VEGETALES EN MÉXICO: PROPUESTAS PARA UNA MEJOR ADAPTACIÓN AL SISTEMA CUPOV
}

\author{
CARLOS ERNESTO ARCUdiA HERNÁNDEZ*
}

\section{INTRODUCCIÓN}

En nuestros días es insoslayable que la revolución industrial tiene un impacto muy importante en la economía internacional. Esa revolución industrial también se deja sentir en la agricultura, en donde ha habido una transformación esencial en la producción. La manipulación de la vida vegetal para servir a la satisfacción de las necesidades humanas no es nueva, encontrándose rastros en la evolución de la cultura humana. La tecnología aplicada en las variedades vegetales está asociada a la investigación científica sobre morfología, anatomía y fisiología vegetal ${ }^{1}$.

La demanda de insumos para la industria agrícola fue satisfecha por vía de las variedades vegetales. Para el botánico, la especie constituye la base del sistema

* Doctor en Derecho Mercantil por la Universidad Complutense de Madrid. Profesor investigador de la Unidad Académica Multidisciplinaria Zona Huasteca de la Universidad Autónoma de San Luis Potosí, México. Investigador nacional nivel 1 del Sistema Nacional de Investigadores del conacyt. Corre-e: carlos.arcudia@uaslp.mx Autor de diversos artículos y capítulos de libro, entre los que destacan "El Poder Judicial en México" (en AA.vv., Estructuras de Administración de Justicia en Estados Compuestos), "La élite del poder económico en México" (Revista de Economía Crítica), "La participación de los inversores institucionales en el gobierno de las grandes sociedades" (Revista de la Facultad de Contaduría y Administración de la UNAM) y "Normas comunes a los sistemas de administración de la Sociedad Anónima Europea" (Boletín Mexicano de Derecho Comparado).

Fecha de recepción: 14 de abril de 2015. Fecha de aceptación: 2 de mayo de 2015. Para citar el artículo: Arcudia Hernández, C. E. El régimen de protección jurídica de las obtenciones vegetales en México: propuestas para una mejor adaptación al sistema cupov. Revista La Propiedad Inmaterial n. ${ }^{\circ} 19$, Universidad Externado de Colombia, enero-junio, 2015, pp. 89-112. DOI: 10.18601/16571959.n19.05

1. Becerra Ramírez, Manuel, 2009, La propiedad industrial en transformación, México, Porrúa, pp. 90-91. 
taxonómico. En el lenguaje ordinario se alude a las plantas por el nombre de su género o especie. El agricultor o el hortelano que cultivan el campo hacen una diferenciación más precisa. No se limitan a plantar patatas o guisantes o a sembrar trigo. Eligen una variedad determinada - una subdivisión de la especie- que prometa mejor calidad o mayor cantidad, u otras ventajas inherentes a las plantas que se cultivan. Si bien las variedades vegetales se conocen desde hace siglos y el término "variedad" se emplea constantemente, no existe una definición precisa del mismo que cuente con la aceptación general ${ }^{2}$.

Ahora bien, y por lo que respecta al tema de nuestro estudio, el régimen jurídico de protección de variedades vegetales, podemos decir que -en generales relativamente reciente, si se lo compara con la larga evolución del sistema de patentes. Este hecho obedece a que los procesos científicos de obtención vegetal comenzaron a desarrollarse a partir del redescubrimiento de las leyes de la herencia a comienzos del siglo xx. Desde entonces la necesidad de protección jurídica de las obtenciones vegetales se basó en lo dilatado del proceso de producción de una nueva variedad, que puede llevar de diez a veinte años. Además el riesgo económico del proceso es muy alto, máxime cuando no existe la seguridad de llegar a producir la variedad deseada y de que una vez obtenida sea un éxito comercial ${ }^{3}$.

A diferencia de lo que sucede en el caso de las patentes, en el sistema de protección de las obtenciones vegetales lo que se protege es la innovación, no la invención, toda vez que se pueden generar plantas nuevas y útiles sin necesidad de extender el conocimiento técnico. Es decir, se protegen únicamente las características físicas que solamente pueden ser incorporadas en un organismo vivo completo, esto es, el fenotipo. El germoplasma de una variedad permanece libre para ser usado en la obtención de variedades nuevas, esta es una forma de describir la información biológica contenida en la innovación ${ }^{4}$. La finalidad del sistema de protección de variedades vegetales es que el obtentor tenga el control sobre la información genética de la variedad, pero garantizando que el público, por su parte, tenga acceso a los demás elementos de la variedad 5 .

El Convenio Internacional para la Protección de Obtenciones Vegetales (CUPOV) fue adoptado en 1961 tras las conferencias diplomáticas celebradas en París en 1957 y 1961. El cupov entró en vigor en 1968, con la ratificación de Alemania, Países Bajos y Reino Unido, siendo modificado en 1972, 1978 y 1991. Este

2. upov, 1982, Información General, Ginebra, upov, p. 6.

3. Cfr. Quintana Carlo, Ignacio, 1975, "La protección de las obtenciones vegetales y la Ley Española de 12 de marzo de 1975", en Actas de Derecho Industrial y Derecho de Autor (ADI), t. 2, pp. 191-192.

4. Funder, John, 1999, "Rethinking patents for plant innovation", en European Intellectual Property Review (EIPR), vol 11, n. ${ }^{\circ} 21$, pp. 554-555.

5. Llewelyn, Margaret, 1997, "The legal protection of Biotechnological inventions", en EIPR, vol. 18, n. ${ }^{\circ} 3$, p. 118. 
Convenio rige los principios del sistema de propiedad industrial a nivel mundial. Los Estados miembros que deseen pertenecer al mismo deben tener su legislación armonizada con el mismo.

En México se adoptó una legislación tipo upov como parte de los compromisos adquiridos por el país con la firma del Tratado de Libre Comercio para América del Norte (TLCAN) de dar protección a las obtenciones vegetales. A mediados de la década de los noventa, el Estado mexicano se adhirió al Acta del cupov de 1978 y promulgó la Ley Federal de Variedades Vegetales (LFVV) para implementar el régimen jurídico de la protección de las obtenciones vegetales. Quizá por la premura en la implementación, la legislación presenta algunas discrepancias con las distintas versiones del cupov.

En las próximas secciones se analizan la adhesión de México al cupov; las características generales del régimen jurídico del cupov, y los cambios experimentados en el régimen jurídico de protección de obtenciones vegetales en las diferentes versiones del mismo. En la última sección se estudia la implementación del cupov en la LFvv. Consideramos que este análisis puede servir de marco para el estudio de la legislación sobre obtenciones vegetales de los países de América Latina.

\section{El Tratado de Libre Comercio para América del Norte y la promulgación de la Ley Federal de Variedades Vegetales}

A partir de los primeros años de la década de 1990 se definieron las acciones del Estado mexicano en materia de liberalización agropecuaria. Entre ellas destacan: la reforma ejidal; la eliminación de los permisos a la importación de alimentos y la negociación del Tratado de Libre Comercio para América del Norte (TLCAN); el desmantelamiento de la Compañía Nacional de Subsistencias Populares; la reducción de subsidios al agro, y la abolición de los programas de extensión agrícola. El proceso de liberalización comercial del agro comenzó entre 1990 y 1991, cuando se eliminaron los permisos a la importación y se redujeron los aranceles. Con el TLCAN se profundizó el proceso de liberalización agropecuaria con Canadá y Estados Unidos, el cual concluyó en $2008^{[6]}$.

Se consideraba desde un comienzo que la liberalización debería elevar la productividad mediante cambios en la estructura productiva: mayor producción de frutas y hortalizas y contracción de la de granos y oleaginosas; menor empleo sectorial total, menos mano de obra y más tierras dedicadas a productos rentables, e intercambio comercial con mayores importaciones de granos y oleaginosas y

6. Yúnez Naude, Antonio, 2010, "Las transformaciones del campo y el papel de las políticas públicas", en Historia económica general de México, México, El Colegio de México, 2010, pp. 729-756. 
crecientes exportaciones ${ }^{7}$. En pocas palabras, se trataba de fomentar la agricultora comercial en detrimento de la agricultura tradicional.

\section{i. i. El Tratado de Libre Comercio para América del Norte}

Como parte de los compromisos contraídos por México en el marco del TLCAN, el artículo 1709, párrafo 3, inciso C) de dicho documento contiene una disposición -esencialmente idéntica a la que aparece en el artículo 27.3 b) de los Acuerdos de Propiedad Industrial relacionados con el Comercio (ADPIC) - que obliga a los países miembros a otorgar protección a las variedades de plantas mediante patentes, un esquema efectivo de protección sui generis o ambos.

Además el inciso a) del Anexo 1701.3 del TlCAN establece: "México realizará su mayor esfuerzo por cumplir lo antes posible con las disposiciones sustantivas de la Convención upov 1978 o 1991, y lo hará antes del término de dos años a partir de la fecha de firma de este Tratado".

Y el inciso b) del mismo anexo dispone: "México aceptará, a partir de la fecha de entrada en vigor de este Tratado, solicitudes de los obtentores de vegetales para variedades de todos los géneros y especies y concederá la protección conforme a tales disposiciones sustantivas con prontitud luego de cumplir con lo que se señala en el inciso a)".

\section{I.2. Adhesión de México Al Cupov de 1978 y promulgación de la LFVV}

Con fecha 12 de diciembre de 1995, el presidente Zedillo firmó el decreto por el que se aprueba el cupov de 1961, revisado en 1972 y 1978 . Este decreto fue publicado en el Diario Oficial de la Federación el 27 de diciembre de 1995, supuestamente para su debida observancia en términos de lo previsto en el artículo 89 , fracción I, de la Constitución.

El 25 de octubre de 1996 fue publicada en el Diario Oficial de la Federación la LFVV. Siguiendo la técnica legislativa europea, la ley separa materias y legisla exclusivamente sobre el derecho del obtentor o creados. Otras legislaciones americanas tratan conjuntamente en una misma norma tanto el derecho del obtentor como la preceptiva sobre comercialización de semillas ${ }^{8}$ (Chile, Uruguay y Argentina $)^{9}$. En México, la comercialización de semillas se encuentra regulada

7. Puyana, Alicia y Romero, José, 2009, México, de la crisis de la deuda al estancamiento económico, México, El Colegio de México, pp. 191-192.

8. Gattari, Carlos y Durante, Martha, 1997, "Comentarios sobre la Ley Federal de variedades vegetales de los Estados Unidos Mexicanos", en Temas de Derecho Industrial y de la Competencia n. ${ }^{\circ}$ 2, p. 299.

9. Cfr. ibíd., p. 301. 
en una ley distinta de la LFVv: la Ley de Producción, Certificación y Comercio de Semillas de $2007^{[10]}$.

En punto a su estructura hay que reseñar que la LFVV contiene cuarenta y ocho artículos, distribuidos en seis títulos. El Título Primero se refiere a las disposiciones generales; el Título Segundo, a la protección de los derechos del obtentor de variedades vegetales; el Título Tercero establece el Comité Calificador de Variedades Vegetales; el Título Cuarto regula el Registro Nacional de Variedades Vegetales; el Título Quinto establece los procedimientos administrativos correspondientes, y el Título Sexto enumera las infracciones por violación a la ley, consignando las multas que en su caso serán aplicables.

En punto a su contenido, la LFVV refleja los principios fundamentales del Acta del cupov de 1978. No obstante es una ley verdaderamente endeble en el aspecto coercitivo. En concreto, no contiene mecanismos de aplicación coercitiva, ni para el resarcimiento de daños y perjuicios ocasionados por la violación de la misma ${ }^{11}$.

Antes de proceder al análisis del régimen jurídico de la protección de variedades vegetales en la LFVV se expondrán las líneas maestras contenidas en las tres actas del CupOv. Este análisis permitirá valorar los aciertos de la LFVV y los aspectos que necesitan adecuarse para que el sistema de protección de obtenciones vegetales opere correctamente.

\section{El Convenio Internacional para la Protección de las Obtenciones Vegetales}

Los derechos de obtención vegetal son un tipo de protección especial, adoptada en las legislaciones nacionales a mediados del siglo pasado y regulada a nivel mundial por el cupov. Este sistema se adapta a las necesidades de los obtentores tradicionales que, mediante una serie de cruces entre variedades similares, diseñan una variedad que presenta determinadas características valiosas de los ascendientes y que además es estable y homogénea. Esta clase de actividad, que claramente contiene la intervención de expertos en la materia, es más una innovación que una invención, siendo esta última objeto del sistema de patentes.

\section{I. Las Actas del Cupov}

Por iniciativa de la ASSINSEL y del gobierno francés se convocó una conferencia diplomática para analizar las diversas opciones de protección de las variedades

10. Publicada en el Diario Oficial de la Federación (Dof) el 15 de junio de 2007 y en vigor desde el 14 de agosto de 2007, en virtud de lo dispuesto en el artículo primero transitorio.

11. Becerra Ramírez, Manuel, 1998, "La Ley Mexicana de Variedades Vegetales", en Liber ad Honorem Sergio Garcia Ramirez, México, unam, pp. 133-134. 
vegetales, conferencia que se reunió en París en diciembre de 1961. El preámbulo del Convenio establece la preocupación de los Estados contratantes por fomentar el desarrollo de variedades vegetales, mediante su protección, y por salvaguardar los intereses de los obtentores. Después de algunas discusiones se concluyó que lo que había que proteger era toda la planta, y no solamente su método de reproducción. Se definió así como objeto de protección la variedad vegetal, al igual que los requisitos a cumplir para acceder a la protección ${ }^{12}$.

\subsubsection{Modificaciones de 1972 y 1978}

En el Acta del cupov de 1972 únicamente se modificaron los artículos 22 y 26 en punto a las mayorías que se requieren para aprobar la reducción de cuotas a determinados países miembros de la Unión Internacional para la Protección de las Obtenciones Vegetales (UPOV) y para permitir a los morosos en sus cuotas seguir gozando del derecho de voto.

En el Acta del cupov de 1978 se eliminó la definición de variedad vegetal contenida en el Acta del cupov de 1961, se emitió una recomendación a los miembros del Convenio para que extendiesen el alcance de la protección a las obtenciones vegetales, haciendo uso de la facultad contenida en el artículo 5.4 del cupov, y se modificó la prohibición de la doble protección para permitir la incorporación de Estados Unidos. En ese país, desde 1930 se conceden patentes a las nuevas variedades vegetales -reproducibles asexualmente- bajo el Plant Patent Act ${ }^{13}$.

\subsubsection{La modificación de 1991}

En respuesta a las demandas para adaptar el sistema CuPOV a las circunstancias actuales de la industria de la obtención vegetal, se convocó una conferencia diplomática en marzo de 1991.

El alcance de la protección de los derechos de los obtentores era muy limitado en el Acta del cupov de 1978, ya que solamente se refería al material de reproducción o multiplicación vegetativa de la variedad protegida, por lo cual no se extendía al producto final ${ }^{14}$. Las otras partes de la planta permanecían libres de uso aunque

12. Nuez Viñals, Fernando et al., 1998 Los Derechos de Propiedad de las Obtenciones Vegetales, Madrid, Ministerio de Agricultura Pesca y Alimentación, pp. 76 y 77.

13. Esta norma está contenida en la sección 35 del United States Code, epígrafes 161 (patentes para plantas), 162 (descripción a efectos de conceder la protección), 163 (ámbito de protección) y 164 (colaboración del Departamento de Agricultura).

14. Sánchez Gil, Olga, 1996, "La ley española de protección de obtenciones vegetales, a la luz de la última reforma del Convenio upov de 19 de marzo de 1991", en ADI XVII, p. 223. 
fuesen muy valiosas. Esto redujo la efectividad de la protección conferida por los derechos de obtención vegetal y fue considerado como un argumento a favor de la patentabilidad de los vegetales. Este argumento, según el cual la protección por derechos de obtención vegetal no era tan efectiva como la de la patente, se vio reforzado por el hecho de que solamente se infringía el derecho de obtención con la venta sin autorización del material de reproducción. El mismo tuvo dos efectos: en primer lugar, la producción de variedades esencialmente derivadas no constituía una infracción; en segundo lugar, un agricultor podría volver a sembrar el producto de la cosecha obtenida a partir de la semilla protegida, sin infringir el derecho de propiedad sobre la variedad vegetal ${ }^{15}$.

También fueron severamente cuestionados el alcance limitado de la protección del derecho de obtentor y su severa limitación mediante el privilegio del agricultor. En esta misma tesitura fue severamente criticada la prohibición de la doble protección ${ }^{16}$.

Para efectos del presente trabajo se analizan aspectos concretos del régimen jurídico de la protección de las variedades vegetales, a saber: el concepto de variedad vegetal; la prohibición de la protección acumulada por patente y título de obtención vegetal; los requisitos para obtener la protección; y, el contenido del derecho del obtentor. Se dejan para un trabajo posterior su agotamiento, las excepciones, y la estructura administrativa encargada de aplicar el régimen jurídico, entre otros temas de importancia.

\subsection{Concepto de variedad vegetal}

El objeto de protección de los derechos de las obtenciones son las variedades vegetales. Este término tiene un significado coloquial: es sinónimo de tipo o especie. En el área de la obtención vegetal el término variedad tiene un significado más especializado que puede ser usado con relativa flexibilidad. Para los científicos, el término variedad no abarca ningún taxón botánico y, por lo tanto, carece de precisión científica para efectos taxonómicos.

El Acta del cupov de 1961 contenía una definición de variedad vegetal según la cual la palabra variedad se aplicaba a cualquier variedad comercial, clon, línea, cepa o híbrido que cumpla con los requisitos de homogeneidad y estabilidad. Este párrafo fue removido en la reforma de 1978, de suerte que no existió una definición legal hasta que se incluyó otra en el Acta de 1991. Los obtentores estuvieron de acuerdo con tal remoción porque únicamente les interesaban los criterios de

15. Llewelyn, Margaret, 1997, “The legal protection of biotechnological inventions", ob. cit., p. 118 .

16. Ibíd. 
distinción, uniformidad y estabilidad, cualquiera que fuese el objeto de la protección legal ${ }^{17}$. La definición que se adoptó en el Acta de 1991 fue la siguiente:

Se entenderá por "variedad" un conjunto de plantas de un solo taxón botánico del rango más bajo conocido que, con independencia de si responde o no plenamente a las condiciones para la concesión de un derecho de obtentor, pueda:

- definirse por la expresión de los caracteres resultantes de un cierto genotipo o de una cierta combinación de genotipos,

- distinguirse de cualquier otro conjunto de plantas por la expresión de uno de dichos caracteres por lo menos,

- considerarse como una unidad, habida cuenta de su aptitud a propagarse sin alteración.

Según Greengrass, esta definición se adoptó a propuesta de los expertos en el campo de las patentes, toda vez que los obtentores la consideraban innecesaria. Esta definición permite interpretar la prohibición del artículo 53 b) del Convenio de la Patente Europea (CPE) y excluir de la patentabilidad a las líneas celulares. La diferencia entre las definiciones del cupov de 1961 y 1991 radica en que la primera era un sinónimo de variedad protegible, mientras que la nueva definición hace una distinción entre el concepto de variedad y el de variedad protegible. A este respecto resulta ilustrativo que se incluye la expresión "con independencia de si responde o no plenamente a las condiciones para la concesión de un derecho de obtentor" 18 .

La conveniencia de mantener esta definición ha sido blanco de críticas, teniendo en cuenta que su eliminación en el Acta de 1978 no habría acarreado mayores dificultades a los obtentores ${ }^{19}$. Es posible que en esta decisión hayan influido los casos en los que la Oficina Europea de Patentes (OEP) interpreta la excepción de patentar vegetales del artículo 53 b) del CPE. Este precepto excluye de la patentabilidad a las variedades vegetales, las razas animales y los procedimientos esencialmente biológicos para la obtención de plantas o animales.

La Cámara de Recursos Técnica de la oep, mediante Decisión de 26 de julio de 1983, resolvió el caso Ciba Geigy y estableció que la prohibición de patentar

17. Crespi, Stephen, 1992, "Patents and Plant Variety Rights: Is there an Interface Problem?", en International Review of Intellectual Property and Competition Law (IIC) vol. 23, n.o 2, pp. 175-176

18. Art. 1 vii) Cupov 1991.

19. Cfr. Greengrass, Barry, 1991, “The 1991 Act of the upov Convention”, en EIPR vol. 13 , p. 467. 
variedades vegetales contenida en el citado artículo 53 b) debía ser interpretada estrictamente. Esto es, que no podía ser extendida a todas las plantas, sino solamente a las variedades vegetales. A este respecto, la OEP estableció que la definición de variedad vegetal del artículo 53 b) es la del Acta del cupov de 1961, llegando a estimar que las excepciones legales a dicho artículo deben interpretarse restrictivamente. Por tanto, serán patentables en el campo de la botánica los siguientes elementos: partes de plantas, los cultivos de tejidos, las líneas celulares de plantas; y en general, todo lo no comprendido en la definición de variedad vegetal en el sentido del Acta de $1961^{[20]}$.

La Cámara de Recursos Técnica de la oep, en su Decisión de 21 de febrero de 1995, resolvió el caso Plant Genetic Systems (PGs). En este asunto, la oep dio un giro de 180 grados en la doctrina adoptada en el caso Ciba Geigy y confirmada en el caso Lubrizo ${ }^{21}$, para negar la patentabilidad de invenciones biotecnológicas en cuya realización estuviera involucrada una variedad vegetal.

En la Decisión de 13 de octubre de 1997, la Cámara de Recursos Técnica de la OEP resolvió el caso Novartis y estableció el criterio para distinguir entre una invención patentable y una no patentable, cuando se refiera a una variedad vegetal. Este criterio ha pasado a la legislación comunitaria, por vía de la Directiva 98/44 sobre Invenciones Biotecnológicas. En el caso Novartis, la Cámara concluye que una reivindicación en la que no se reclama individualmente una variedad vegetal específica no está excluida de la patentabilidad en virtud del artículo 53 b) del CPE, incluso cuando la misma puede comprender variedades vegetales. En el razonamiento se explica que a la hora de determinar el objeto de una reivindicación importa definir en qué medida la invención a patentar es una invención genérica o bien una invención concreta. Si es genérica puede ser patentable, ya que se realizaría tanto en variedades vegetales como en cualquier tipo de vegetales; el hecho de que la misma se pueda aplicar a variedades vegetales no quiere decir que se excluya de la patentabilidad ${ }^{22}$.

En sendas resoluciones de fecha 25 de marzo de 2015, la Cámara de Recursos Técnica de la oep estableció -en el caso G 02/12 Tomates II- que la prohibición de patentar procedimientos esencialmente biológicos para la obtención de plantas o animales no afecta las reivindicaciones de patentes sobre plantas o partes de plantas que a la fecha de la solicitud solamente puedan ser obtenidas por esos procedimientos. En el caso G 02/13 Brocoli II, la Cámara estableció que una reivindicación de producto (sobre plantas o material vegetal que no sea una

20. Cfr. Lema Devesa, Carlos, 1999, "Las patentes sobre biotecnología en el Derecho Español y en el Derecho Comunitario Europeo", en ADI xx, pp. 225 y 226.

21. Cfr. ibíd., p. 226.

22. Botana Agra, Manuel, 2000, "Precisiones de la Alta Cámara de Recursos de la OEP sobre la prohibición de patentar vegetales", en $A D I$ XXI, p. 195. 
variedad vegetal) definido a través de un procedimiento (“product-by-process") no es inadmisible por el mero hecho de que las características relativas al proceso de obtención definan un procedimiento esencialmente biológico para la producción de plantas $^{23}$.

\subsection{LA PROHIBICIÓN DE LA DOBLE PROTECCIÓN}

Esta prohibición tiene sus orígenes en la Primera Conferencia de París de 1957, en la cual los expertos analizaron la posibilidad de conceder protección a los desarrollos de los obtentores por medio de patente o de un título de protección especial. No les pareció viable la posibilidad de que se otorgara una protección acumulada de dos títulos de protección sobre una misma creación. Por lo tanto, la doble protección se excluyó explícitamente en el párrafo segundo del artículo 2.1 del Acta de 1961, y no se le dio mayor importancia al tema.

En este sentido, es realmente sorprendente que en los documentos preparatorios de la Conferencia Diplomática para la Protección de Nuevas Variedades Vegetales entre mayo de 1957 y noviembre de 1961 no se encontraran bases objetivas para la prohibición. El grupo de expertos jurídicos consideraba que debía concederse solo un tipo de protección para la misma especie o grupo de especies. La cuestión fue abordada en la Conferencia Diplomática, y la Delegación francesa del BIRPI sostuvo la coexistencia de ambos sistemas, con un tipo de protección diferente y sin que compitieran entre sí. Finalmente se adoptó la postura de permitir a los Estados miembros la libre elección del sistema de protección, con la restricción de que no se podrían conceder a la misma especie los dos tipos de protección. Como se desprende, no existe consideración objetiva alguna en particular para esta prohibición. En descargo de los autores del Convenio, se puede decir que en aquel momento no podían haber previsto el desarrollo exponencial de las ciencias naturales ${ }^{24}$.

Sin embargo, la prohibición de la doble protección fue modificada en el Acta de 1978 para permitir el acceso de Estados Unidos al Convenio. Desde esa época no existe homogeneidad entre los miembros del cupov ${ }^{25}$.

23. Cfr. Resolución G 02/12 de la Alta Cámara de Recursos Técnica de la oep de 25 de marzo de 2015 a la apelación promovida por el Ministerio de Agricultura de Israel en contra de Unilever; y, Resolución G 02/13 de la Alta Cámara de Recursos Técnica de la oep de 25 de marzo de 2015 a la apelación promovida por Plant Biosciences Limited en contra de Syngenta Participations AG, Groupe Limagrain Holding.

24. Cfr. Straus, Joseph, 1984, "Patent Protection for New Varieties of Plants Produced by Genetic Engineering - Should 'Double Protection' be Prohibited?", en IIC vol. 15, n. ${ }^{\circ} 4$, pp. $439-440$.

25. Se añadió el artículo 37.1 que establecía: "Sin perjuicio de lo dispuesto en el Artículo 2.1, todo Estado que, antes de la expiración del plazo durante el que la presente Acta está abierta a la firma, prevea la protección bajo las diferentes formas mencionadas en el 
En el Acta de 1991 esta prohibición fue suprimida, dejando a los Estados la posibilidad de proteger una variedad con dos títulos diferentes de protección.

\subsection{Requisitos PARA ObTENER LA PROTECCIÓN DE UNA VARIEDAD VEGETAL}

\subsubsection{Novedad}

A diferencia de lo que ocurre en la legislación de patentes, la novedad consiste en que la variedad no debe haber sido comercializada con anterioridad a la fecha de solicitud de protección de la variedad; sin embargo, se establece como obligatorio un año de período de gracia. El artículo 6.1 (b) del Acta de 1978 establecía que la variedad no debía ser ofrecida o vendida en el mercado.

En el Acta de 1991 se habla de que el material de propagación no debe haberse vendido o estar en poder de terceros con el consentimiento del obtentor, con propósitos de explotación. Así pues, se pretende englobar determinadas actividades que, como no tienen un propósito comercial, no destruyen la novedad; por ejemplo, trabajar con líneas endogámicas para producir un tercer híbrido ${ }^{26}$.

\subsubsection{Distintividad}

Una variedad debe ser claramente distinguible de otras en el momento de la solicitud de protección. En el Acta de 1991 se suprimió el adjetivo “importante” de las características que debían distinguir a la variedad, contenido en el Acta de 1978. De este modo, se evitó la ambigüedad de dicho término, ya que se interpretaba como si lo que distinguiese a una variedad de otra debía ser de mucho mérito ${ }^{27}$.

\subsubsection{Homogeneidad}

La variedad deberá ser suficientemente homogénea, teniendo en cuenta las particularidades que presente su reproducción sexuada o su multiplicación vegetativa.

Artículo 2.1 para un mismo género o una misma especie, podrá continuar previéndola si, en el momento de la firma de la presente Acta o de la presentación de su instrumento de ratificación, aceptación o aprobación de la presente Acta o de adhesión a ésta, notifica ese hecho al Secretario General". Cfr. Straus, Joseph, 1987, “The Relationship Between Plant Variety Protection and Patent Protection for Biotechnological Inventions form International View Point", en IIC vol. 18, n. ${ }^{\circ}$ 6, p. 729; Straus, Joseph, 1984, "Patent Protection for New Varieties of Plants", ob. cit., p. 440.

26. Greengrass, Barry, 1991, "The 1991 Act of the upov Convention", ob. cit., p. 468.

27. Ibíd., p. 468. 


\subsubsection{Estabilidad}

La variedad deberá ser estable en sus caracteres esenciales, es decir, deberá permanecer conforme a su definición después de reproducciones o multiplicaciones sucesivas, o cuando el obtentor haya definido un ciclo particular de reproducciones o de multiplicaciones, al final de cada ciclo.

\subsection{Contenido de la protección del título de obtentor}

La protección consiste en un derecho de propiedad exclusiva para el obtentor o creador de la variedad. Derecho exclusivo que se materializaba, en las actas de 1961 y 1978, en la exigencia de la autorización previa del titular para la producción y venta u oferta en venta del material de reproducción de la variedad protegida ${ }^{28}$.

Esta protección tenía dos límites: el privilegio del obtentor, que le otorga libertad para utilizar la variedad protegida para desarrollar nuevas variedades; y el privilegio del agricultor, que otorga libertad a los agricultores para utilizar en su propia explotación el material de reproducción obtenido de la cosecha de la variedad protegida ${ }^{29}$.

Durante la revisión de 1978, a pesar de que tanto las asociaciones de obtentores (Assinsel, Ciopora) como los abogados (AIPpI) habían solicitado, en las reuniones previas, extender la protección de las obtenciones vegetales, solamente se logró una Recomendación de la Conferencia Diplomática, en la que se instó a los Estados miembros a extender la protección para salvaguardar los legítimos intereses de los obtentores, pero en la práctica esa recomendación fue letra muerta ${ }^{30}$.

En el Acta de 1991 se extendieron los actos para los que se requiere autorización del titular. Así las cosas, el titular de la variedad debe dar su consentimiento para la producción, el acondicionamiento con fines de propagación, el ofrecimiento en venta, la comercialización, la importación, la exportación y el almacenamiento del material de propagación para los fines anteriores ${ }^{31}$.

28. Cfr. Verma, Surinder, 1995, "trips and Plant Variety Protection in Developing Countries" en EIPR vol. 16, n. ${ }^{\circ}$ 6, p. 284, y Greengrass, Barry, 1989, "Upov and the Protection of Plant Breeders - Past Developments, Future Perspectives", en IIC vol 20, n. ${ }^{\circ}$ 5, p. 624.

29. El privilegio del obtentor se establece de forma clara en el artículo 5.3 del cupov de 1961 y el del agricultor se infiere del artículo 5.1 del cupov de 1961 ya que la autorización del obtentor se requiere únicamente para los actos enunciados en dicho apartado que tengan por objeto la comercialización de la variedad.

30. Straus, Joseph, 1987, "The Relationship Between Plant Variety Protection", ob. cit. p. 729 .

31. Nuez Viñals et al., "Los Derechos de Propiedad de las Obtenciones Vegetales", ob. cit., p. 77; Greengrass, "The 1991 Act of the upov Convention", ob. cit., p. 469. 
También se previó la posibilidad de extender los derechos del obtentor hasta el material cultivado, incluyendo plantas enteras o partes de las mismas, si han sido producidas violando los derechos del obtentor. Se autorizó incluso a los Estados miembros a extender el derecho de exclusiva hasta los productos elaborados a partir del material cultivado, si el titular del derecho no tuvo oportunidad de ejercerlo antes de que los productos estuviesen en el mercado ${ }^{32}$.

Otra extensión de la protección contenida en el Acta de 1991 es la adopción del concepto de variedad esencialmente derivada. En el sistema upov una variedad protegida puede ser utilizada libremente para crear otra. Sucede que, en más de una ocasión, variedades protegidas eran modificadas por medio de lo que (conforme a la tecnología inglesa) se conoce como cosmetic breeding y protegidas a través de un nuevo título de obtención vegetal, a favor de la persona que realizaba la modificación ${ }^{33}$.

En el Acta de 1991 se atajó esta situación incluyendo el concepto de variedad esencialmente derivada, que no puede ser explotada sin autorización del obtentor de la variedad originaria ${ }^{34}$. De acuerdo con el artículo 14.5 b) del cupov una variedad es esencialmente derivada de otra cuando:

i) se deriva principalmente de la variedad inicial, o de una variedad que a su vez se deriva principalmente de la variedad inicial, conservando al mismo tiempo las expresiones de los caracteres esenciales que resulten del genotipo o de la combinación de genotipos de la variedad inicial;

ii) se distingue claramente de la variedad inicial, y

iii) salvo por lo que respecta a las diferencias resultantes de la derivación, es conforme a la variedad inicial en la expresión de los caracteres esenciales que resulten del genotipo o de la combinación de genotipos de la variedad inicial.

Una vez expuestas las líneas generales del régimen de protección de obtenciones vegetales contenido en las diversas actas del cupov, se procede al análisis de su implementación en la LFVV.

32. Verma, "trips and Plant Variety Protection", ob. cit., p. 284; Correa, Carlos, 1992, "Biological Resources and Intellectual Property Rights", en EIPR vol. 14, n. ${ }^{\circ}$ 5, p. 156, y LLEWELYN, “The legal protection of Biotechnological Inventions", ob. cit., p. 119.

33. También se podían obtener variedades esencialmente derivadas por selección de las mutaciones naturales o inducidas de una variedad; la selección de variantes individuales de la variedad inicial o su transformación por ingeniería genética. Cfr. VERMA, "TRIPS and Plant Variety Protection", ob. cit., p. 285.

34. Cfr. Greengrass, "The 1991 Act of the upov Convention”, ob. cit., p. 470; Verma, "Trips and Plant Variety Protection", ob. cit., p. 285, y Correa, "Biological resources and intellectual property rights”, ob. cit., p. 155. 


\section{RÉGimen JuRÍdico de La PROTECCIÓN DE LAS OBTENCiONES vegetales en la Ley Federal de Variedades Vegetales}

\section{3.i. Concepto de variedad vegetal}

Si bien el Acta de 1978 del cupov eliminó la definición de variedad vegetal, la LFVV define a la variedad vegetal como una subdivisión de una especie que incluye a un grupo de individuos con características similares y que se considera estable y homogénea.

En materia taxonómica se habla de organismo con un gran número de características comunes, al momento de ubicarlos en una determinada categoría o subcategoría de acuerdo con la jerarquía que hay que seguir para una conveniente clasificación. De acuerdo con estos planteamientos, el concepto de variedad vegetal de la LFVV quedaría expresado aludiendo a una subespecie que incluye un grupo de organismos con características similares que se considera estable y homogénea ${ }^{35}$.

Pues bien, a nuestro modo de ver la inclusión del concepto de variedad vegetal en la LFVV es un acierto, aunque el Acta de 1978 no establezca tal concepto. La adopción de esta medida se justifica porque solo estableciendo el concepto de variedad vegetal se puede determinar la frontera entre el sistema de patentes y el sistema de títulos de obtención vegetal.

En todo caso, no debe olvidarse que la definición de variedad vegetal de la LFVV omite dos aspectos importantes de la definición del Acta de 1991, a saber: no distingue entre los conceptos de variedad vegetal y variedad vegetal protegible. En efecto, la definición de variedad vegetal del Acta de 1991 contiene la precisión de que "con independencia de si responde o no plenamente a las condiciones para la concesión de un derecho de obtentor". Esta precisión no es gratuita: su razón de ser consiste en evitar que variedades vegetales que no cumplan con los requisitos para acceder a la protección mediante título de obtención vegetal puedan ser protegidas mediante patente, así como en garantizar la prohibición de patentar variedades vegetales.

Por otra parte, la mencionada definición también omite la referencia (contenida en el Acta de 1991) de que los caracteres que definen a una variedad vegetal son resultantes de un genotipo o de una cierta combinación de genotipos. Como se expuso, esta exigencia del cupov persigue llevar al plano del genotipo la caracterización vegetal en lugar de hacerlo al del fenotipo ${ }^{36}$.

35. Rangel Ortiz, Horario, 1998, "La protección de las variedades vegetales en el Derecho Mexicano", en $A D I$ XIX, p. 143.

36. La identificación de una variedad vegetal por medio de su genotipo es un criterio más preciso que la identificación por la simple apariencia externa de una planta, y es más acorde con el estado actual de la biotecnología. 
Por ende, consideramos conveniente modificar la fracción ix del artículo 2 de la LFVV para sustituir el concepto de variedad vegetal por el concepto de variedad vegetal del Acta de 1991.

\subsection{Prohibición de la doble protección}

México, miembro del Acta de 1978 del cupov, incluye esta prohibición en el artículo 16 de la Ley de Propiedad Industrial (LPI). Pues bien, llegados a este punto es necesario realizar algunas observaciones sobre la prohibición de la doble protección en el derecho mexicano. En primer lugar, la LPI no hace referencia al concepto de variedad vegetal. En este sentido, sería recomendable que la LPI plasmase el concepto de variedad vegetal, contenido en la fracción Ix del artículo 2 de la LFVV.

En segundo lugar; aunque la LPI hiciera referencia al concepto de variedad vegetal de la fracción IX del artículo 2 de la LFVv, las deficiencias que presenta esta definición ${ }^{37}$ pueden dar lugar a que una variedad vegetal que no cumpla con los requisitos que exige la LFVV pueda ser patentada. Consideramos que este es un ulterior motivo para que la LFvV incorpore el concepto de variedad vegetal del Acta de 1991.

\subsection{Requisitos}

El artículo 7 de la LFVV prevé el cumplimiento de cuatro requisitos sustantivos como condición para tener acceso a la protección a través del título de obtentor. La LFVV exige que en la variedad vegetal que se pretende proteger a través del título de obtentor estén presentes las siguientes condiciones: novedad, distintividad, estabilidad y homogeneidad.

\subsubsection{Novedad}

En el régimen de la LFVV la novedad es equivalente a la ausencia de comercialización de la variedad vegetal a proteger. En efecto, el artículo 7, fracción I, incisos a) y b) dispone que una variedad se tendrá por nueva cuando a la fecha de presentación de la solicitud el material de reproducción no haya sido enajenado en más de un año anterior a esa fecha en el territorio nacional, o en cualquier otro país por más de seis años en el caso de árboles y vides, y de cuatro años para el resto de las especies.

37. Concretamente, la definición de variedad vegetal de la LFVV no distingue entre variedad vegetal y variedad protegible. 
El Comité Calificador de Variedades Vegetales (instancia encargada del examen de los requisitos) investigará si la variedad vegetal se ha comercializado fuera de los plazos que señala la fracción I del artículo 7 de la LFvV, y realizará consultas en los registros oficiales de los organismos internacionales y de los países con los que hubiere convenios sobre la materia; asimismo, se difundirán por los medios que se consideren idóneos, los datos y características de la variedad vegetal para que sean del conocimiento público.

Llegados a este punto es necesario puntualizar que el Acta de 1991 introdujo algunas modificaciones en relación con el Acta de 1978 respecto de los hechos destructores de la novedad. Así, en el Acta de 1978 los actos que conllevan ofrecimiento de venta o comercialización de la variedad quiebran la novedad. En el Acta de 1991 el ofrecimiento en venta no queda comprendido entre los actos destructores de la novedad. Por el contrario, queda comprendida la venta o la entrega a terceros del material de propagación de la variedad para la explotación de tal variedad.

Por otra parte, el Acta de 1978 preveía la posibilidad de que la ley nacional estableciera (de manera opcional) un plazo de gracia de un año respecto de actos comerciales sobre las variedades anteriores a la fecha de la solicitud. Este plazo ha devenido obligatorio bajo el Acta de 1991. Así pues, fácilmente se puede apreciar que el requisito de la novedad de la LFvV se aproxima más a las exigencias del del Acta de 1991 que a las del Acta de $1978^{[38]}$.

\subsubsection{Distintividad}

Tendrá esta característica la variedad vegetal que se distinga técnica y claramente por uno o varios caracteres pertinentes respecto de cualquiera otra variedad, cuya existencia sea conocida en el momento en que se solicite la protección. Dichos caracteres deberán reconocerse y describirse con precisión. El reglamento señalará las diversas referencias para determinar si una variedad es o no conocida ${ }^{39}$.

El artículo 2, fracción I del Reglamento de la Ley Federal de Variedades Vegetales (RLFVV) establece que los caracteres pertinentes son expresiones fenotípicas y genotípicas propias de la variedad vegetal, que permiten su identificación; pero no hace la precisión de qué se entiende por conocimiento previo de la variedad vegetal

El artículo 31 del RLfvv faculta al Comité de Calificación de Variedades Vegetales para verificar el requisito de distinción, considerando cualquier carac-

38. En Iberoamérica, además de la legislación mexicana, contemplan este plazo obligatorio las de Brasil y Chile y la Decisión 345 de la Comunidad Andina. Cfr. Correa, Carlos, 2001, "El Convenio upov. Una perspectiva latinoamericana”, en Temas de Derecho Industrial y de la Competencia n. ${ }^{\circ}$ 5, p. 143.

39. Artículo 7, fracción II de la LFVv. 
terística que pueda ser determinada y descrita con precisión como distintiva, de manera que la variedad vegetal pueda diferenciarse de otras sin dificultad alguna, con independencia de la naturaleza de los caracteres pertinentes señalados en el informe técnico.

Los caracteres pertinentes que se utilicen para distinguir una variedad vegetal podrán ser cualitativos y cuantitativos. En ambos casos, la variación se definirá mediante niveles de expresión fenotípica en función de las necesidades de distinción que, para los que no sean mesurables, será de tipo discontinuo y para los cuantificables, continuo entre dos extremos, los cuales se describirán en las guías técnicas respectivas o en las normas oficiales mexicanas.

Del proceso de revisión, investigación o consulta que realice el Comité deberá acreditarse que la variedad vegetal se distingue cuando menos en un carácter pertinente de otras variedades vegetales protegidas o del dominio público.

\subsubsection{Homogeneidad}

Tendrá esta característica la variedad vegetal que sea suficientemente uniforme en sus caracteres pertinentes a reserva de la variación ${ }^{40}$.

\subsubsection{Estabilidad}

Tendrá esta característica la variedad vegetal que conserve inalterados sus caracteres pertinentes después de reproducciones o propagaciones sucesivas ${ }^{41}$.

Como se puede observar, ninguno de los preceptos citados toma en cuenta el caso de las variedades vegetales híbridas que no pueden ser reproducidas sucesivamente, sino que tienen un ciclo particular de reproducciones o de multiplicaciones. Para estos casos tanto el inciso d) del artículo 6 del Acta de 1978 como el artículo 9 del Acta de 1991 establecen que la variedad híbrida será estable si permanecen inalterados sus caracteres pertinentes al final de cada ciclo particular de reproducciones o de multiplicaciones.

\section{4. Ámbito de la protección}

De modo similar a como ocurre en materia de derecho de autor, la LFVV se refiere a dos manifestaciones del derecho de los obtentores: una de carácter moral y otra de carácter económico.

40. Artículo 7, fracción IV de la LfvV.

41. Artículo 7, fracción iII de la LFvv. 
Artículo 4 de la LFVV. Los derechos que la LFVV otorga a los obtentores son los siguientes:

I. Ser reconocido como obtentor de una variedad vegetal. Este derecho es inalienable e imprescriptible, $y$

II. Aprovechar y explotar, en forma exclusiva y de manera temporal, por sí o por terceros con su consentimiento, una variedad vegetal y su material de propagación, para su producción, reproducción, distribución o venta, así como para la producción de otras variedades vegetales e híbridos con fines comerciales. Estos derechos tendrán una duración de:

a) Dieciocho años para especies perennes (forestales, frutícolas, vides, ornamentales) y sus portainjertos, $y$

b) Quince años para las especies no incluidas en el inciso anterior. Estos plazos se contarán a partir de la fecha de expedición del título de obtentor y, una vez transcurridos, la variedad vegetal, su aprovechamiento y explotación, pasarán al dominio público.

De los preceptos anteriormente expuestos fácilmente se deduce que la LFVV se sitúa en un punto intermedio entre las actas del cupov de 1978 y de 1991. En efecto, la LFVV concede al obtentor el derecho exclusivo de producir la variedad vegetal, cualquiera que sea su fin, así como el monopolio de la distribución de la variedad vegetal y su comercialización. Por el contrario, no le concede la exclusiva para la preparación para la reproducción o la comercialización, la oferta en venta, la exportación ni la importación. En este sentido, consideramos que la LFVV debe incluir todos los actos contemplados en el Acta de 1991, sobre todo la importación y la exportación, que han sido fuente de controversia.

Por otra parte, cabe poner de relieve una "originalidad" de la LFVV. La fracción I del artículo 4 de la LFVV (de forma similar a los derechos de autor) establece que la persona que desarrolle una variedad vegetal tiene el derecho (inalienable e imprescriptible) de ser reconocida como tal. No sabemos si esta "ocurrencia" del legislador mexicano responde a su afán de reconocer el esfuerzo de los mejoradores de variedades vegetales, o bien a una confusión del derecho de las obtenciones vegetales con el derecho de autor ${ }^{42}$. En todo caso, esta disposición parece estar fuera de lugar: bastaría que el nombre de la persona que desarrolló la variedad se consigne en el título de obtención vegetal.

42. En efecto, las actas del cupov de 1961/1972, 1978 y 1991 contienen este "derecho moral" a ser reconocido como obtentor de la variedad. El motivo de esta ausencia es que el obtentor busca obtener provecho económico de su innovación, más que ser reconocido como el obtentor de la variedad vegetal protegible. 
La LFVV no contiene reglamentación del concepto de variedad esencialmente derivada, situación que consideramos juega en contra de los intereses nacionales. En efecto, un país como México, rico en biodiversidad y que realiza esfuerzos en mejora vegetal clásica, se ve perjudicado con la adopción de un sistema de protección que se basa únicamente en el Acta del cupov de 1978, que no contempla la derivación esencial. Concretamente, las variedades nacionales serán susceptibles de competencia por parte de los obtentores que modifiquen superficialmente las variedades existentes. En cambio, si la LFVV incorpora el concepto de variedad esencialmente derivada, las variedades modificadas de forma superficial no podrían ser explotadas sin la autorización del obtentor de la variedad inicial ${ }^{43}$.

\subsection{Obligaciones del obtentor}

El artículo 18 de la LFVV dispone que toda persona que use o aproveche la variedad vegetal para cualquier propósito está obligada a utilizar y respetar la denominación aprobada, y cuando esta se utilice junto con una marca o nombre comercial u otra indicación, deberá ser fácilmente reconocible y distinguible, indicando la genealogía y el origen de la variedad.

Según el artículo 19 de la LFVv, los derechos que confiere el título de obtentor podrán gravarse y transmitirse total o parcialmente, salvo el derecho moral de paternidad. El obtentor o su causahabiente están obligados a otorgar licencias de emergencia cuando la explotación de una variedad vegetal se considere indispensable para satisfacer las necesidades básicas de un sector de la población y exista deficiencia en la oferta o en el abasto. Por otra parte, en caso de que la variedad vegetal no se hubiere explotado en un plazo de tres años contados a partir de la fecha de expedición del título de obtentor, se procederá como si fuere de emergencia, en términos del artículo 25 de la LFVV.

Sin embargo, en todos los casos lo que habilita la declaración de emergencia es la necesidad nacional de "producto" y no de la "variedad", ya que es suplir el mercado de producto (como material de consumo e industria y no como medio de propagación) lo que efectivamente llega al público usuario y puede constituir emergencia nacional, comportando una licencia imperativa ${ }^{44}$.

43. En Iberoamérica, la Decisión 345 de la Comisión del Acuerdo de Cartagena prevé que el derecho del obtentor de impedir que terceros realicen determinados actos sin su consentimiento, se extienda a las variedades esencialmente derivadas. El concepto de variedad esencialmente derivada también se encuentra presente en la legislación de obtenciones vegetales de Brasil. Cfr. Correa, "El Convenio upov", ob. cit., p. 144.

44. Gattari y Durante, "Comentarios sobre la Ley Federal de Variedades Vegetales", ob. cit., p. 312. 


\subsection{GÉNEROS y ESPECIES PROTEGIDAS}

En el Acta del cupov de 1961 se obligaba a los Estados miembros a conceder protección a cinco géneros o especies botánicas. En el Acta de 1978, se obligaba a los Estados miembros a proteger un mínimo de cinco géneros, y esta protección debería extenderse a un mínimo de 24 géneros o especies en un período de ocho años posterior a su ingreso a la UPov.

En el artículo 4 del Acta de 1978 se exhortaba a los Estados miembros a extender progresivamente la protección a un número cada vez mayor de géneros y especies botánicos, pero en ningún caso se les exhortaba a la protección de todo el reino vegetal ${ }^{45}$.

Por contraste con la situación descrita, el artículo 3 del Acta de 1991 establece como obligación de los Estados miembros el otorgar protección a todos los géneros y especies del reino vegetal en un período de cinco años a partir de la entrada en vigor de dicha acta. A los nuevos Estados miembros se les concede un plazo de 10 años para proteger todos los géneros y especies ${ }^{46}$.

Ahora bien, en México - miembro del Acta de 1978- se extendió la protección a todos los géneros y especies botánicos sin utilizar los plazos de gracia que a tal efecto concede el Acta de 1978. En efecto, el artículo 4.2 de esta Acta establece que los Estados miembros se comprometen a adoptar las medidas necesarias para aplicar progresivamente las disposiciones del cupov al mayor número de géneros y especies botánicos. Seguidamente, el artículo 4.3 declara que, a la entrada en vigor del cupov en el territorio de un Estado, este deberá aplicar las disposiciones del Convenio a un mínimo de cinco géneros o especies; en un plazo de tres años, esta protección deberá extenderse a un mínimo de diez géneros o especies; a los seis años, deberá extenderse a un mínimo de dieciocho géneros o especies; y, por último, a los ocho años la protección deberá abarcar veinticuatro géneros o especies, por lo menos.

Esta "celeridad" del legislador mexicano, tendiente a proteger todos los géneros y especies pudo obedecer al cumplimiento de los compromisos del TLCAN que, como se ha visto, obligaba a México a proteger todos los géneros y especies botánicos en un plazo de dos años a partir de la entrada en vigor del Tratado.

45. Verma, “trips and Plant Variety Protection”, ob. cit., p. 283, y Greengrass, “The 1991 Act”, ob. cit., p. 468.

46. Ibíd. 


\section{Conclusiones}

De lo anteriormente expuesto cabe concluir cuanto sigue.

México adoptó el cupov y promulgó su LFVv en 1996 como consecuencia del compromiso asumido por nuestro país en el TLCAN de aplicar las disposiciones sustantivas de las actas de 1978 o 1991 del cupov y de otorgar protección para todos los géneros y especies botánicos. La LFVV refleja satisfactoriamente la instrumentación de las principales disposiciones del régimen legal previsto en el Acta del cupov de 1978 en materia de protección para las variedades u obtenciones vegetales. Esto es aplicable en particular a temas tales como las condiciones para la protección, examen y verificación de las condiciones de la protección, duración de la protección y contenido del derecho.

El cupov regula el régimen jurídico de protección de obtenciones vegetales a nivel internacional. Fue adoptado en 1961 y ha sido modificado en 1972, 1978 y 1991. Esta última modificación cambió radicalmente el sistema Upov. Las principales causas de la reforma de 1991 fueron: la insuficiente regulación en las versiones anteriores del cupov, toda vez que ofrecía una protección mucho menor a la del sistema de patentes y había una serie de actos que no estaban contemplados en el ámbito de la protección y comprometían seriamente los derechos del obtentos; y, el desarrollo de la biotecnología, ya que con los avances en de esta ciencia el sistema de protección de obtenciones vegetales quedó reservado para las variedades obtenidas por métodos tradicionales.

El concepto de variedad vegetal, el objeto de protección del sistema upov, ha variado a través de las distintas versiones. En el Acta de 1961 se esbozaba una definición descriptiva del concepto de variedad vegetal. En el Acta de 1978 se eliminó la definición, pero esto trajo cierta inconformidad con los miembros que prohíben la protección acumulada por patente y título de obtención, ya que sirve para delimitar ambos sistemas. En el Acta de 1991 se retoma y se hace referencia a genotipo y fenotipo de la planta, así como a su nivel en la escala taxonómica.

La prohibición de la doble protección ha sido uno de los temas que más ha generado discusión en el seno de la upov. Los países europeos prohíben la protección acumulada por patente y título de obtención vegetal. Estados Unidos, en cambio, otorga protección por medio de patente desde 1930. El Acta de 1961 contenía esa prohibición. En el Acta de 1978 se dejó abierta la posibilidad de que los países que antes de adherirse al cupov no la tuvieran en sus legislaciones, la pudieran mantener. En el Acta de 1991 se eliminó, si bien tampoco se obligó a conceder la protección acumulada.

De los requisitos para obtener la protección -novedad, distintividad, homogeneidad y estabilidad-, los que han sufrido cambios en las diferentes actas del cupov son los de novedad y distintividad. En efecto, la novedad -entendida como ausencia de comercialización- experimentó una ampliación de los efectos 
destructivos de la misma en el Acta de 1991 para englobar actos que si bien no constituyen comercialización directa, sí tienden a ello. En punto a la distintividad se eliminó el adjetivo "importante" que modificaba al sustantivo características de las cuales debería diferenciarse de otras variedades.

El contenido y alcance de la protección experimentó una ampliación considerable. En efecto, en las actas del cupov anteriores a la de 1991 se exigía la autorización del titular para la producción y venta u oferta en venta del material de propagación del material de reproducción de la variedad protegida. En el Acta de 1991 se requiere autorización del titular de la variedad para la producción, el acondicionamiento con fines de propagación, el ofrecimiento en venta, la comercialización, la importación, la exportación y el almacenamiento del material de propagación para los fines anteriores. También se prevé la extensión del alcance hasta el producto de la cosecha del material de la variedad si no se pudo ejercer con anterioridad el derecho de exclusiva. En el plano del desarrollo de nuevas variedades, se adoptó el concepto de variedad esencialmente derivada para evitar la apropiación indebida de variedades mediante modificaciones leves.

La LFVV contiene un concepto de variedad vegetal que, si bien no es obligatorio porque México pertenece al Acta de 1978, omite distinguir entre el concepto de variedad vegetal y el de variedad vegetal protegible. En efecto, la definición de variedad vegetal del Acta de 1991 contiene la precisión de que "con independencia de si responde o no plenamente a las condiciones para la concesión de un derecho de obtentor". Por otra parte, omite la referencia a que los caracteres que definen a una variedad vegetal son resultantes de un genotipo o de una cierta combinación de genotipos. Y si bien nuestra legislación de patentes prohíbe la patentabilidad de variedades vegetales, no hace referencia al concepto de la LFVV, lo cual garantizaría un adecuado deslinde de ambos sistemas de protección.

En cuanto a los requisitos para obtener la protección, la LFVV se ajusta más al Acta de 1991 que a la de 1978 en torno al requisito de la novedad. En el de la distintividad se establece que la diferenciación deberá ser por caracteres "pertinentes", concepto que introduce incertidumbre jurídica, la cual se trató de eliminar del cupov con la supresión de los "caracteres importantes". El requisito de la estabilidad no contempla el caso de las variedades híbridas.

En punto al ámbito de protección del título de obtención vegetal, la LFVV se sitúa en un punto intermedio entre las actas de 1978 y de 1991 del cupov. En efecto, la LFVV concede al obtentor el derecho exclusivo de producir la variedad vegetal, cualquiera que sea su fin, así como el monopolio de la distribución de la variedad vegetal y su comercialización. Por el contrario, no le concede la exclusiva para la preparación para la reproducción o la comercialización, la oferta en venta, la exportación ni la importación. En este sentido, consideramos que la LFVV debe incluir todos los actos contemplados en el Acta del cupov de 1991, sobre todo la importación y la exportación, que han sido fuente de controversia. Por otra 
parte, se debe poner de relieve una "originalidad" de la LFVV: la fracción I del artículo 4 -de forma similar a lo que ocurre con los derechos de autor- establece que la persona que desarrolle una variedad vegetal tiene el derecho (inalienable e imprescriptible) de ser reconocida como tal. No sabemos la razón de esta "ocurrencia" del legislador mexicano. La protección se extiende a todos los géneros y especies botánicos, como en el Acta de 1991 del cupov, y la LFVV no contempla el concepto de variedad esencialmente derivada.

\section{BiBLIOGRAFÍA}

Becerra Ramírez, Manuel, 1998, "La Ley Mexicana de Variedades Vegetales”, en Liber ad Honorem Sergio García Ramírez, México, unam.

Becerra Ramírez, Manuel, 2009, La propiedad industrial en transformación, México, Porrúa.

Botana Agra, Manuel, 2000, "Precisiones de la Alta Cámara de Recursos de la OEP sobre la prohibición de patentar vegetales", en Actas de Derecho Industrialy de Derecho de Autor (ADI) xxI, pp. 1195-1200.

Correa, Carlos, 1992, "Biological Resources and Intellectual Property Rights", en European Intellectual Property Review (EIPR) vol. 14, n. ' 5, pp. 154-157.

Correa, Carlos, 2001, "El Convenio upov. Una perspectiva latinoamericana”, en Temas de Derecho Industrial y de la Competencia n. ${ }^{\circ}$ 5, pp. 141-167.

Crespi Stephen, 1985, "Patent protection in biotechnology: Questions, answers and observations", en Biotechnology and Patent Protection an International Review, París, ocDe, pp. 36-86.

Crespi Stephen, 1992, "Patents and Plant Variety Rights: Is there an Interface Problem?", en International Review of Intellectual Property and Competition Law (IIC), vol. 23, n. ${ }^{\circ}$, pp. 168-184.

Gattari, Carlos y Durante, Martha, 1997, "Comentarios sobre la Ley Federal de variedades vegetales de los Estados Unidos Mexicanos”, en Temas de Derecho Industrial y de la Competencia n. ${ }^{\circ}$, pp. 299-316.

Greengrass, Barry, 1991, “The 1991 Act of the upov Convention”, en EIPR, vol. 13, pp. 466-472.

Greengrass, Barry, 1989, "upov and the Protection of Plant Breeders - Past Developments, Future Perspectives", en IIC, vol 20, n. ${ }^{\circ}$ 5, pp. 622-636.

Lema Devesa, Carlos, 1999, "Las patentes sobre biotecnología en el Derecho Español y en el Derecho Comunitario Europeo”, en ADI xx, pp. 219-235.

Llewelyn, Margaret, 1997, “The legal protection of biotechnological inventions”, en European Intellectual Property Review (EIPR), vol. 18, n. ${ }^{\circ} 3$, pp. 115-127. Nuez Viñals, Fernando et al., 1998, Los Derechos de Propiedad de las Obtenciones Vegetales, Madrid, Ministerio de Agricultura Pesca y Alimentación. 
Puyana, Alicia y Romero, José, 2009, México, de la crisis de la deuda al estancamiento económico, México, El Colegio de México.

Quintana Carlo, Ignacio, 1975, "La protección de las obtenciones vegetales y la Ley Española de 12 de marzo de 1975”, en $A D I$ t. 2, pp. 189-263.

Rangel Ortiz, Horario, 1998, "La protección de las variedades vegetales en el Derecho Mexicano", en ADI XIX, pp. 127-167.

SÁnchez Gil, Olga, 1996, "La ley española de protección de obtenciones vegetales, a la luz de la última reforma del Convenio upov de 19 de marzo de 1991 ”, en $A D I$ XVII, pp. 219-259.

Sánchez Gil, Olga, 1984, "Patent Protection for New Varieties of Plants Produced by Genetic Engineering - Should 'Double Protection' be Prohibited?", en IIC vol. 15, n.o 4, pp. 426-442.

Straus, Joseph, 1987, “The Relationship Between Plant Variety Protection and Patent Protection for Biotechnological Inventions for Biotechnological Inventions from International View Point”, en IIC vol. 18, n. ${ }^{\circ}$ 6, pp. 723-737. Under, JoHn, 1999, "Rethinking patents for plant innovation”, en European Intellectual Property Review (EIPR), vol. 11, n.o 21, pp. 551-577.

upov, 1982, Información General, Ginebra, upov.

Verma, Surinder, 1995, “Trips and Plant Variety Protection in Developing Countries”, en EIPR vol. 16, n. ${ }^{\circ}$ 6, pp. 281-289.

Yúnez Naude, Antonio, 2010, "La transformaciones del campo y el papel de las políticas públicas", en Historia económica general de México, México, El Colegio de México, pp. 729-756. 